\title{
Quality of life questionnaire predicts poor exercise capacity only in HFpEF and not in HFrEF
}

Artan Ahmeti ${ }^{1,2}$, Michael Y. Henein ${ }^{3,4}$, Pranvera Ibrahimi ${ }^{1,3}$, Shpend Elezi ${ }^{2}$, Edmond Haliti ${ }^{1,2}$, Afrim Poniku ${ }^{1,2}$, Arlind Batalli ${ }^{1,2}$ and Gani Bajraktari ${ }^{1,2,3^{*}}$ (i)

\begin{abstract}
Background: The Minnesota Living with Heart Failure Questionnaire (MLHFQ) is the most widely used measure of quality of life (QoL) in HF patients. This prospective study aimed to assess the relationship between QoL and exercise capacity in HF patients.
\end{abstract}

Methods: The study subjects were 118 consecutive patients with chronic HF (62 \pm 10 years, 57 females, in NYHA I-III). Patients answered a MLHFQ questionnaire in the same day of complete clinical, biochemical and echocardiographic assessment. They also underwent a 5 min walk test (6-MWT), in the same day, which grouped them into; Group I: $\leq$ $300 \mathrm{~m}$ and Group II: >300 m. In addition, left ventricular (LV) ejection fraction (EF), divided them into: Group A, with preserved EF (HFpEF) and Group B with reduced EF (HFrEF).

Results: The mean MLHFQ total scale score was $48( \pm 17)$. The total scale, and the physical and emotional functional MLHFQ scores did not differ between HFpEF and HFpEF. Group I patients were older $(p=0.003)$, had higher NYHA functional class $(p=0.002)$, faster baseline heart rate $(p=0.006)$, higher prevalence of smoking $(p=0.015)$, higher global, physical and emotional MLHFQ scores $(p<0.001$, for all), larger left atrial (LA) diameter $(p=0.001)$, shorter LV filling time $(p=0.027)$, higher E/e' ratio $(0.02)$, shorter isovolumic relaxation time $(p=0.028)$, lower septal $a^{\prime}(p=0.019)$ and $s^{\prime}(p=0.023)$, compared to Group II.

Independent predictors of 6-MWT distance for the group as a whole were increased MLHFQ total score $(p=0.005)$, older age $(p=0.035)$, and diabetes $(p=0.045)$, in HFpEF were total MLHFQ $(p=0.007)$ and diabetes $(p=0.045)$ but in HFrEF were only LA enlargement $(p=0.005)$ and age $(p=0.013$. A total MLHFQ score of 48.5 had a sensitivity of $67 \%$ and specificity of 63\% (AUC on ROC analysis of 72\%) for limited exercise performance in HF patients.

Conclusions: Quality of life, assessment by MLHFQ, is the best correlate of exercise capacity measured by 6-MWT, particularly in HFpEF patients. Despite worse ejection fraction in HFrEF, signs of raised LA pressure independently determine exercise capacity in these patients.

Keywords: Heart failure, The Minnesota Living with Heart Failure Questionnaire, Exercise capacity, 6 min walk test, Echocardiography, Quality of life

\footnotetext{
* Correspondence: gani.bajraktari@uni-pr.edu; gani.bajraktari@umu.se

${ }^{1}$ Clinic of Cardiology, University Clinical Centre of Kosova, Rrethi i Spitalit, P.N, 10000 Prishtina, Kosovo

${ }^{2}$ Medical Faculty, University of Prishtina, Prishtina, Kosovo

Full list of author information is available at the end of the article
} 


\section{Background}

Heart failure (HF) represents end stage heart disease irrespective of the underlying etiology, and is acknowledged as a major cause of mortality and morbidity $[1,2]$. Exercise intolerance and impaired quality of life (QoL) reflect poor prognosis in HF patients, and are considered the hallmark of disease severity, irrespective of left ventricular (LV) ejection fraction (EF) [3-7]. Treatment of $\mathrm{HF}$ aims at improving the clinical status, the functional capacity and QoL, as well as reducing mortality and hospitalizations [8]. Recently, QoL improvement has been shown as one of the most important treatment goals in HF, particularly with the documented increase in life expectancy $[9,10]$. Moreover, bearing in mind the expected short life expectancy in these patients, QoL seems to be an important objective that needs to always be addressed [11-13].

The QoL in HF is commonly assessed by the Minnesota Living with Heart Failure questionnaire (MLHFQ) [14] and the Quality of Life with Heart Failure questionnaire (QLHF) $[15,16]$. We have translated these two questionnaires into Albanian language and used them in Kosovo Heart Failure Patients [17]. The relationship between QoL and other demographic parameters proved controversial with some studies showing that older age is associated with lower QoL and others failing to show similar relationships [18-21]. Also, the relationship between QoL and other parameters, such as gender and race, remain controversial [22, 23]. Few studies investigated the relationship of QoL with exercise capacity and breathlessness, which showed that QoL correlated with limited exercise and higher NYHA class [24, 25]. But, in those studies the relationship of QoL and LV EF remains not certain [25-27]. Therefore, the aim of this prospective study was to assess the relationship between MLHFQ and exercise capacity in HF patients.

\section{Methods}

\section{Study population}

We studied 118 consecutive patients with a clinical diagnosis of congestive HF (age $62 \pm 10$ years, 57 female) with ischemic or non-ischemic aetiology, who were in New York Heart Association (NYHA) functional class I-III, and were referred to the Clinic of Cardiology, University Clinical Centre of Kosovo, between December 2014 and September 2016. At the time of the study all patients were on full cardiac medications, optimized at least 2 weeks prior to enrollment. Patients with NYHA class IV, those with limited physical activity due to factors other than cardiac symptoms (e.g. arthritis), with more than mild renal or hepatic failure, with chronic obstructive pulmonary disease, with recent acute coronary syndrome, stroke, psychological or psychiatric disorders, or those with severe anemia, were excluded from the study. All patients signed a written informed consent to participate in the study, which was approved by the Ethics Committee of the Medical Faculty, University of Prishtina. This study was supported and monitored by Kosovo Society of Cardiology [27], which is trying to implement European Society of Cardiology guidelines and other current diagnostic and therapeutic recommendations.

\section{Data collection}

A detailed history and clinical assessment were obtained in all patients. Routine biochemical tests, including hemoglobin, lipid profile, blood glucose level and kidney function, were also performed in all study patients. Estimated body mass index (BMI) was calculated from weight and height measurements. Waist and hip measurements were also made and waist/hip ratio was calculated.

\section{Quality of life assessment}

The MLHFQ contains 21 questions, whose aim is to determine how HF affects the physical, psychological and socioeconomic conditions of the patients (Additional file 1 Table S1). The questions refer to the signs and symptoms of HF, social relationships, physical and sexual activity, work and emotions [14] and assesses how HF affected the patient's life during the previous month. The MLHFQ has a scoring range of 0 for no impairment to 105 for maximum impairment. The questions cover symptoms and signs relevant to HF, physical activity, social interaction, sexual activity, work, and emotions. Three scores were determined: an overall score (21 items, 0-105), the physical dimension (8 items, 0-40), and the emotional dimension (5 items, 0-25), with the highest scores reflecting the worse QoL. The scale of answers to each question ranges from 0 (none) to 5 (very much), where 0 represented no limitation and 105 represented maximal limitation.

\section{Echocardiographic examination}

A single operator performed all echocardiographic examinations using a Philips Intelligent E-33 system with a multi-frequency transducer, and harmonic imaging as appropriate. Using conventional landmarks and recommendations of the American Society of Echocardiography and European Association of Echocardiography $[28,29]$ we obtained all measurements including, interventricular septal (IVS) thickness, posterior wall (PW) thickness, and LV dimensions, LV volumes and EF using the modified Simpson's method and left ventricular mass (LVM) using Devereux formula [30].

Ventricular long axis motion was also studied using conventional methods previously described [31], from which the following measurements were obtained; total amplitude as the mitral annular plane systolic excursion (MAPSE) and the tricuspid plane systolic excursion (TAPSE), and long axis myocardial velocities in systole 
$\left(\mathrm{s}^{\prime}\right)$, early (e') and late (a') diastole. Mean value of the lateral and septal e' velocities was also calculated. LV diastolic function was assessed from spectral Doppler recordings, from which LV early (E wave), late (A wave) diastolic velocities, E/A ratio and E/e' (mean lateral and septal) ratio were all calculated. Finally, LV isovolumic relaxation time (IVRT) was measured. LV filling pattern was considered 'restrictive' when E/A ratio was $>2.0$, E wave deceleration time $<140 \mathrm{~ms}$ and the LA trasverse diameter was $>40 \mathrm{~mm}$ [33]. LA diameter and volumes were measured, according to the guidelines of the American Society of Echocardiography and European Association of Echocardiography [29], maximal volume (LAV max) at the end systole and LA minimal volume (LAV min) at end diastole. LA total emptying fraction was calculated using the formula [32]:

$$
\begin{aligned}
& \text { LA total emptying fraction } \\
& =\text { LAV max-LAV min/LAV } \max x 100
\end{aligned}
$$

\section{Measurements of LV dyssynchrony}

Indirect assessment of LV dyssynchrony was obtained by measuring total isovolumic time (t-IVT), Tei Index and LV-RV pre-ejection time delay, a spreviously described [33] using total LV filling time and ejection times. Total isovolumic time (t-IVT) was calculated as 60 - (total ejection time + total filling time) and was expressed in $\mathrm{s} / \mathrm{min}$ [34]. Tei index was calculated as the ratio between t-IVT and ejection time [35].

Mitral and tricuspid regurgitation severity were assessed by colour and continuous wave Doppler and was graded as mild, moderate, or severe according to the relative jet area to that of the left atrium (LA) in line with the recommendations of the American Society of Echocardiography [36]. Retrograde trans-tricuspid pressure drop $>35 \mathrm{mmHg}$ was taken as an evidence for pulmonary hypertension [28]. All M-mode and Doppler recordings were made at a fast speed of $100 \mathrm{~mm} / \mathrm{s}$ with a superimposed ECG (lead II). From the pulmonary artery flow recordings pulmonary artery acceleration time (PAAT) [37]. The LV outflow tract (LVOT) diameter and area were measured [38] in order to calculate the average velocity time integral (VTI) and the stroke volume (SV) [39].

\section{NT-pro BNP measurement}

Blood was taken from an antecubital vein in the morning, sober and after staying extended for $20 \mathrm{~min}$. Blood samples were collected into tubes containing potassium ethylenediaminetetraacetic acid (EDTA) (1 g/L plasma) and $\mathrm{N}$-terminal proBNP were calculated with the Cobas Elecsys E411 analyzer (range 5-35,000 pg/mL) using chemiluminescent immunoassay kit (Roche Diagnostics, Grenach - Wyhlen, Germany).

\section{Six minute walk test}

Within $24 \mathrm{~h}$ of the echocardiographic examination a 6MWT was performed on a level hallway surface and was administered by a specialized nurse blinded to the results of the echocardiogram. According to the method of Gyatt et al. [40] patients were informed of the purpose and protocol of the 6-MWT, which was conducted in a standardized fashion without interrupting patient's regular medications [41]. A $15 \mathrm{~m}$ flat, obstacle-free corridor was used and patients were instructed to walk as far as they can, turning $180^{\circ}$ after they had reached the end of the corridor, during the allocated time of $6 \mathrm{~min}$. Patients walked unaccompanied so as not to influence walking speed. At the end of the 6 min the supervising nurse measured the total distance walked by the patient.

\section{Statistical analysis}

Data are presented as mean \pm SD or proportions (\% of patients). Continuous data was compared with twotailed unpaired Student's $t$ test and discrete data with Chi-square test. Correlations were tested with Pearson coefficients. Predictors of 6-MWT distance were identified with univariate analysis and multivariate logistic regression was performed using the step-wise method, a significant difference was defined as $P<0.05$ (2tailed). Patients were divided according to their ability to walk $>300 \mathrm{~m}$ into good and limited exercise performance groups [42], and were compared using unpaired Student $t$-test.

\section{Results}

The baseline characteristics of the study population are presented in Table 1. All 118 patients completed the MLHFQ. Patients mean age was $62 \pm 9.8$ years, and $48 \%$ were women. The most common comorbidities were hypertension (67\%) and diabetes mellitus (27\%) and 30\% were smokers. Mean 6-MWT distance was $315 \pm 115 \mathrm{~m}$, and $47 \%$ were in NYHA class II. Table 2 presents baseline echocardiographic variables.

The score of total MLHFQ scale was $48 \pm 17$, whereas the physical and emotional MLHFQ subscales scores were $24 \pm 9$ and $9 \pm 5$, respectively (Table 1 ). The total physical and emotional MLHFQ subscale scores were not different in patients with $\mathrm{HF}$ and preserved $\mathrm{EF}$ (HFpEF) compared to those with HF and reduced EF (HFrEF) (Table 2).

Five of $59(10 \%)$ patients with HFpEF had AF, compared to 13 of $59(22 \%)$ patients with $\operatorname{HFrEF}(p=0.07)$. LA diameter was significantly larger in AF patients compared to non-AF patients in HFrEF patients $(p=0.001)$, but not in HFpEF ( $p=0.123)$. However, the 6-MWT distance was not significant in both subgroups. 
Table 1 Baseline characteristics of the study patients

\begin{tabular}{|c|c|}
\hline Variable & Means $\pm S D$ \\
\hline Age (years) & $62 \pm 9.8$ \\
\hline BMI $\left(\mathrm{kg} / \mathrm{m}^{2}\right)$ & $29 \pm 3.8$ \\
\hline Waist/hip ratio & $0.96 \pm 0.1$ \\
\hline HR (beat/min) & $83 \pm 19$ \\
\hline Diabetes mellitus (\%) & 27 \\
\hline Arterial hypertension (\%) & 67 \\
\hline Smoking (\%) & 30 \\
\hline LBBB (\%) & 14 \\
\hline NYHA class I, II, III (\%) & $30,47,23$ \\
\hline Sinus rhythm (\%) & 80 \\
\hline B-blockers (\%) & 78 \\
\hline ACEi (\%) & 81 \\
\hline Diuretic (\%) & 76 \\
\hline Ca-blockers (\%) & 12 \\
\hline Aspirin (\%) & 77 \\
\hline Oral anticoagulants (\%) & 22 \\
\hline 6MWT(m) & $315 \pm 115$ \\
\hline Hemoglobin (g/dL) & $12.6 \pm 1.7$ \\
\hline Creatinine $(\mu \mathrm{mol} / \mathrm{l})$ & $96 \pm 45$ \\
\hline NT-ProBNP (pg/mL) & $3630 \pm 3742$ \\
\hline MLHFQ - total score & $48 \pm 17$ \\
\hline MLHFQ - physical score & $24 \pm 9$ \\
\hline MLHFQ - emotional score & $9 \pm 5$ \\
\hline
\end{tabular}

$B M I$ body mass index, BUN blood urea nitrogen, SBP systolic blood pressure, $D B P$ diastolic blood pressure, HR heart rate, NYHA New York heart association, $A C E i$ angiotensin converting enzyme inhibitors, Ca-blockers Calcium channel blockers, NT-ProBNP N-terminal pro b-type natriuretic peptide, $L B B B$ left bundle branch block, WBC white blood cell, $6 M W T 6$ min walking test, MLHFQ minnesota living with heart failure questionnaire

\section{Patients with limited exercise vs. preserved exercise capacity (Tables 3 and 4 )}

Patients with limited exercise, who walked $<300 \mathrm{~m}$ during 6-MWT, were older $(p=0.003)$, had higher NYHA functional class $(p=0.002)$, faster baseline heart rate ( $p=0.006)$, higher prevalence of smoking ( $p=0.015)$, and higher global, physical and emotional MLHFQ scores $(p<0.001$, for all), compared to those with good exercise capacity. Patients with limited exercise also had larger LA

Table 2 Comparison of quality of life between patients HFpEF and HFrEF

\begin{tabular}{llll}
\hline Variable & $\begin{array}{l}\text { HFpEF } \\
(n=59)\end{array}$ & $\begin{array}{l}\text { HFrEF } \\
(n=59)\end{array}$ & $P$ value \\
\hline MLHFQ total & $47 \pm 18$ & $50 \pm 16$ & 0.328 \\
MFHFQ physical & $23 \pm 9$ & $26 \pm 8$ & 0.066 \\
MFHFQ emotional & $9 \pm 5$ & $9 \pm 4$ & 0.521 \\
\hline
\end{tabular}

MLHFQ minnesota living with heart failure questioners
Table 3 Comparison of clinical and biochemical data between patients with limited exercise vs. preserved exercise capacity (6-min walk distance)

\begin{tabular}{|c|c|c|c|}
\hline Variable & $\begin{array}{l}6 \mathrm{MWT}>300 \mathrm{~m} \\
(n=76)\end{array}$ & $\begin{array}{l}6 \mathrm{MWT}<300 \mathrm{~m} \\
(n=42)\end{array}$ & $P$ value \\
\hline Age (years) & $60 \pm 9.5$ & $66 \pm 9$ & 0.003 \\
\hline Smoking (\%) & 28 & 33 & 0.516 \\
\hline Diabetes (\%) & 20 & 41 & 0.015 \\
\hline Arterial hypertension (\%) & 66 & 69 & 0.839 \\
\hline LBBB (\%) & 10 & 21 & 0.169 \\
\hline Waist/hips ratio & $0.95 \pm 0.1$ & $0.98 \pm 0.1$ & 0.036 \\
\hline BMI $\left(\mathrm{kg} / \mathrm{m}^{2}\right)$ & $29 \pm 4.1$ & $28 \pm 3.8$ & 0.730 \\
\hline $\mathrm{BSA}\left(\mathrm{m}^{2}\right)$ & $1.2 \pm 0.2$ & $1.1 \pm 0.2$ & 0.046 \\
\hline NYHA class & & & $0.002^{\mathrm{a}}$ \\
\hline NYHA class I, II, III (\%) & $38,47,15$ & $14,45,41$ & \\
\hline HFpEF (\%) & 46 & 57 & 0.249 \\
\hline Fasting glucose (mmol/L) & $6.4 \pm 2.5$ & $7.8 \pm 2.9$ & 0.013 \\
\hline Creatinine $(\mu \mathrm{mol} / \mathrm{L})$ & $97.6 \pm 54$ & $93.0 \pm 21$ & 0.604 \\
\hline Hemoglobin (g/dL) & $12.7 \pm 1.6$ & $12.3 \pm 2.0$ & 0.203 \\
\hline HR (beats/min) & $72 \pm 15$ & $80 \pm 13$ & 0.006 \\
\hline NT-ProBNP (pg/mL & $1510 \pm 4146$ & $1832 \pm 2907$ & 0.66 \\
\hline MLHFQ total & $43.8 \pm 16.9$ & $56.9 \pm 14.4$ & $<0.001$ \\
\hline MLHFQ physical & $22.1 \pm 9$ & $27.6 \pm 6.9$ & 0.001 \\
\hline MLHFQ emotional & $8.0 \pm 4.3$ & $10.9 \pm 4.3$ & 0.001 \\
\hline
\end{tabular}

$B M I$ body mass index, BSA body surface area, $H R$ heart rate, NYHA New York heart association, $L B B B$ left bundle branch block, NT-ProBNP N-terminal pro b-type natriuretic peptide, $H F p E F$ heart failure with a preserved ejection fraction, $M L H F Q$ minnesota living with heart failure questionnaire

${ }^{a} \mathrm{NYHA}$ class significance between study groups

diameter $(p=0.001)$, shorter LV FT $(p=0.027)$, smaller septal MAPSE $(p=0.037)$, higher $\mathrm{E} / \mathrm{e}^{\prime}$ ratio $(0.020)$, shorter IVRT $(p=0.028)$, PAAT $(p=0.005)$, lower septal a' $(p=0.019)$ and s' $(p=0.023)$, compared to those with preserved exercise capacity. All other clinical and echocardiographic parameters were not significantly different between two groups.

\section{Relationship of total MLHFQ with clinical, biochemical and echocardiographic variables (Table 5)}

In the patients' group as a whole, total MLHFQ score had strong correlation with 6-MWT distance, lateral s' ( $p<0.001$ for both), good correlation with LVMI $(p=0.001)$ and with lateral MAPSE $(p=0.009)$, and weak correlation with hemoglobin level $(p=0.024)$. In HFpEF, total MLHFQ score had strong correlation with 6-MWT distance ( $<<0.001$, Fig. 1$)$, and weak correlation with lateral s' $(p=0.014)$, LVMI $(p=0.027)$ and with hemoglobin level $(p=0.016)$, whereas in HFrEF patients it has only a weak correlation with lateral s' $(p=0.03)$, LVMI $(p=0.027)$, lateral MAPSE $(p=0.027)$ and with $\mathrm{E} / \mathrm{A}$ ratio $(p=0.047)$. 
Table 4 Comparison of echocardiographic data between patients with limited exercise vs. preserved exercise capacity (6-min walk distance)

\begin{tabular}{|c|c|c|c|}
\hline Variable & $\begin{array}{l}6 \mathrm{MWT}>300 \mathrm{~m} \\
(n=76)\end{array}$ & $\begin{array}{l}6 \mathrm{MWT}<300 \mathrm{~m} \\
(n=42)\end{array}$ & $P$ value \\
\hline LV EF (\%) & $48 \pm 15$ & $45 \pm 15$ & 0.445 \\
\hline IVS (cm) & $1.12 \pm 0.2$ & $1.11 \pm 0.1$ & 0.986 \\
\hline LA diameter (cm) & $4.2 \pm 0.7$ & $4.7 \pm 0.9$ & 0.001 \\
\hline LV EDD (cm) & $5.7 \pm 0.8$ & $5.9 \pm 1.1$ & 0.271 \\
\hline LV ESD (cm) & $4.2 \pm 1.1$ & $4.5 \pm 1.3$ & 0.261 \\
\hline Lateral MAPSE (cm) & $1.3 \pm 0.9$ & $1.2 \pm 1.1$ & 0.786 \\
\hline Septal MAPSE (cm) & $1.0 \pm 0.3$ & $0.9 \pm 0.3$ & 0.037 \\
\hline TAPSE (cm) & $2.2 \pm 2.3$ & $2.1 \pm 2.6$ & 0.670 \\
\hline LV posterior wall $(\mathrm{cm})$ & $1.1 \pm 0.4$ & $1.0 \pm 0.1$ & 0.913 \\
\hline E/A ratio & $1.0 \pm 0.6$ & $1.1 \pm 0.8$ & 0.325 \\
\hline $\mathrm{FT}(\mathrm{ms})$ & $431 \pm 138$ & $379 \pm 105$ & 0.027 \\
\hline IVRT (ms) & $132 \pm 42$ & $111 \pm 34$ & 0.028 \\
\hline PAAT (ms) & $114 \pm 23$ & $100 \pm 22$ & 0.005 \\
\hline E/e' ratio & $10 \pm 4.1$ & $13 \pm 8.0$ & 0.020 \\
\hline Lareral e' (cm/s) & $6.1 \pm 2.5$ & $6.0 \pm 2.7$ & 0.881 \\
\hline Lateral a' (cm/s) & $8.2 \pm 3.7$ & $7.6 \pm 3.5$ & 0.393 \\
\hline Lateral s' (cm/s) & $5.5 \pm 1.5$ & $4.9 \pm 1.6$ & 0.074 \\
\hline Septal e' (cm/s) & $5.1 \pm 2.2$ & $4.6 \pm 2.1$ & 0.295 \\
\hline Septal a' (cm/s) & $7.5 \pm 2.4$ & $6.4 \pm 1.9$ & 0.019 \\
\hline Septal s' (cm/s) & $4.6 \pm 1.6$ & $4.0 \pm 1.1$ & 0.023 \\
\hline LA EF (\%) & $49 \pm 17$ & $45 \pm 16$ & 0.313 \\
\hline
\end{tabular}

$L V$ left ventricle, $E D D$ end-diastolic dimension, $E S D$ end-systolic dimension, $F T$ filling time, ET Ejection time, IVS interventricular septum, IVRT isovolemic relaxation time, e' early diastolic myocardial velocity, $s^{\prime}$ systolic myocardial velocity, $L A$ left atrium, LA EF Left atrial emptying fraction, $A$ atrial diastolic velocity, $E$ early diastolic filling velocity, PAAT pulmonary artery acceleration time, MAPSE mitral annular plane systolic excursion, TAPSE tricuspid annular plane systolic excursion

\section{Predictors of limited 6-MWT distance in HF patients (Table 6)}

\section{Predictors of limited 6 MWT distance in all HF patients}

In univariate analysis, total MLHFQ $(p<0.001)$, physical MLHFQ $(p=0.002)$, emotional MLHFQ $(p=0.002)$, age $(p=0.005)$, diabetes $(p=0.017)$, atrial fibrillation $(p=0.006)$, LA diameter $(p=0.001)$, IVRT $(p=0.047)$, PAAT $(p=0.008)$, septal MAPSE $(\mathrm{p}=0.04)$, E/e' $(p=0.029)$, septal a' $(p=0.033)$, and septal s' $(p=0.041)$, predicted limited 6 MWT distance. In multivariate analysis, only total MLHFQ score $(p=0.005)$, age $(p=0.035)$ and the diabetes $(p=0.045)$ remained independent predictors of limited 6MWT distance. A total MLHFQ score of 48.5 had a sensitivity of $67 \%$ and specificity of $63 \%$ (AUC on ROC analysis of $72 \%$ ) for predicting limited exercise performance (Fig. 2).

\section{Predictors of limited 6-MWT distance in HFpEF patients} Univariate analysis identified total MLHFQ ( $p=0.001)$, physical MLHFQ ( $p=0.026)$, emotional MLHFQ $(p=0.007), \operatorname{BSA}(p=0.009)$, diabetes $(p=0.036)$, and NYHA class $>1(p=0.012)$, hemoglobin level $(p=0.039)$, increased LVMI $(p=0.023)$, low lateral s' $(p=0.013)$ and a' $(p=0.032)$ as predictors of limited 6-MWT distance. In multivariate analysis, total MLHFQ $(p=0.007)$ and diabetes $(p=0.045)$ independently predicted the limited 6-MWT distance.

\section{Predictors of limited 6 MWT distance in HFrEF patients}

In univariate analysis, physical MLHFQ $(p=0.044)$, age $(p=0.015)$, NYHA class $>1(p=0.036), \mathrm{LV}$ mass $(\mathrm{p}=0.036)$ and LA diameter $(p=0.008)$, predicted the 6-MWT limited exercise distance. In multivariate analysis, only LA enlargement $(p=0.005)$ and age $(p=0.013)$ remained independent predictors of limited 6-MWT distance.

\section{Discussion}

\section{Findings}

The results of this study analysis can be summarized as follows: 1) the total scale, physical and emotional MLHFQ subscale scores were not different between HFpEF and HFrEF patients. 2) Patients with limited exercise capacity were older, had higher NYHA functional class, faster baseline heart rate, higher prevalence of smoking and higher global, physical and emotional MLHFQ scores, compared to those with good exercise capacity. 3) Patients with limited exercise capacity, also had larger LA, shorter LV FT, worse longitudinal systolic function and raised LV filling pressures, compared to those with preserved exercise capacity. 4) Total MLHFQ score had strong correlation with 6-MWT distance in the patients group as a whole and in HFpEF subgroup, but not in HFrEF. 5) Total MLHFQ score, age and diabetes were the only independent predictors of limited 6-MWT distance in the whole group of patients and in HFpEF subgroup. It was LA enlargement and age which independently predicted limited exercise capacity in HFrEF.

\section{Results interpretation}

MLHFQ irrespective of its components; physical or emotional seems to be a good measure of exercise capacity, since it correlated strongly with the 6-MWT distance in the HF group irrespective of EF. Thus, it could be used to reflect the overall cardiac status, when used to evaluate patients' response to treatment. It however, does not reflect the underlying cardiac structural or functional disturbances, which contribute to the limited exercise capacity in individual patients, and which might need different treatments. Age seemed to be correlating with limited exercise capacity but nothing can be done about it. On the other hand baseline heart rate proved to be an equally important factor but can be managed by beta blockers [43] or other forms of heart rate controlling medications e.g. Ivabridine [44], or the combination of the two [45]. 
Table 5 Correlation of MLHFQ total score in HF patients with clinical, biochemical and echocardiographic variables in study patients

\begin{tabular}{|c|c|c|c|c|c|c|}
\hline \multirow[t]{2}{*}{ Variable } & \multicolumn{2}{|c|}{ All study patients $(n=118)$} & \multicolumn{2}{|c|}{ HFpEF $(n=59)$} & \multicolumn{2}{|c|}{$\operatorname{HFrEF}(n=59)$} \\
\hline & $\mathrm{R}$ & $\mathrm{p}$ & r & P & $\mathrm{R}$ & $\mathrm{P}$ \\
\hline 6-MWT & -0.359 & $<0.001$ & -0.500 & $<0.000$ & -0.203 & 0.123 \\
\hline Age & 0.081 & 0.281 & 0.013 & 0.922 & 0.144 & 0.278 \\
\hline Creatinine & -0.076 & 0.418 & 0.093 & 0.481 & -0.178 & 0.181 \\
\hline Hemoglobin & -0.208 & 0.024 & -0.312 & 0.016 & -0.136 & 0.304 \\
\hline BMl & 0.119 & 0.198 & 0.179 & 0.175 & 0.073 & 0.582 \\
\hline BSA & -0.139 & 0.134 & -0.171 & 0.194 & -0.104 & 0.433 \\
\hline LVMI & 0.292 & 0.001 & 0.289 & 0.027 & 0.287 & 0.027 \\
\hline LA diameter & 0.112 & 0.226 & 0.108 & 0.415 & 0.070 & 0.598 \\
\hline LV EF & -0.081 & 0.384 & 0.117 & 0.378 & -0.179 & 0.174 \\
\hline$E / A$ & 0.165 & 0.080 & -0.092 & 0.489 & 0.269 & 0.047 \\
\hline Lateral MAPSE & -0.245 & 0.009 & -0.160 & 0.229 & -0.308 & 0.021 \\
\hline Lateral s' & -0.306 & $<0.001$ & -0.319 & 0.014 & -0.282 & 0.030 \\
\hline$E / e^{\prime}$ & 0.173 & 0.092 & 0.026 & 0.855 & 0.263 & 0.089 \\
\hline Septal s' & -0.137 & 0.179 & -0.111 & 0.426 & -0.094 & 0.544 \\
\hline
\end{tabular}

MLHFQ minnesota living with heart failure questionnaire, 6-MWT 6 min walking test, BMI body mass index, BSA body surface area, EDD end-diastolic dimension, MAPSE mitral annular plane systolic excursion, $A$ atrial diastolic velocity, $E$ early diastolic filling, velocity, $e^{\prime}$ early diastolic myocardial velocity, $s$ ' systolic myocardial velocity, LVMI left ventricular mass index

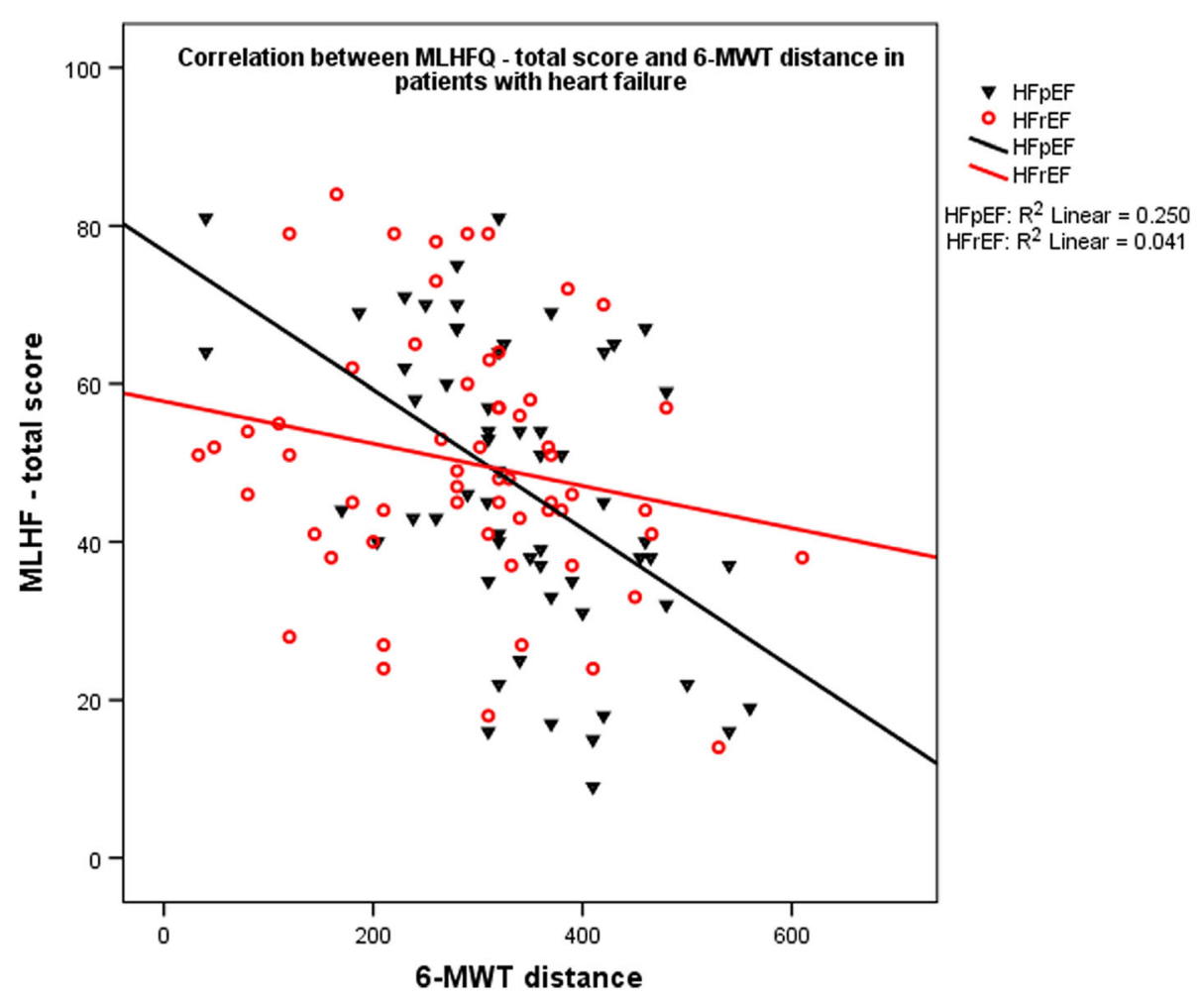

Fig. 1 Correlation between total MLHFQ score and 6-MWT distance in patients with heart failure 
Table 6 Predictors of limited exercise in HF patients

\begin{tabular}{|c|c|c|c|c|c|c|}
\hline \multirow[t]{2}{*}{ Variable } & \multicolumn{3}{|c|}{ Univariate predictors } & \multicolumn{3}{|c|}{ Multivariate predictors } \\
\hline & OR & $\mathrm{Cl} 95 \%$ & $P$ value & $\mathrm{OR}$ & Cl 95\% & $P$ value \\
\hline \multicolumn{7}{|l|}{ Whole HF study patients } \\
\hline MLHFQ - total score & 1.053 & $(1.025-1.081)$ & $<0.001$ & 1.080 & $(1.023-1.140)$ & 0.005 \\
\hline MLHFQ - physical score & 1.085 & $(1.031-1.141)$ & 0.002 & & & \\
\hline MLHFQ - emotional score & 1.163 & $(1.060-1.277)$ & 0.001 & & & \\
\hline Age & 1.069 & $(1.021-1.120)$ & 0.005 & 1.101 & $(1.007-1.203)$ & 0.035 \\
\hline Gender & 0.576 & $(0.269-1.232)$ & 0.155 & & & \\
\hline Diabetes mellitus & 2.765 & $(1.199-6.379)$ & 0.017 & 4.876 & $(1.037-22.94)$ & 0.045 \\
\hline LA diameter & 2.500 & $(1.467-4.260)$ & 0.001 & & & \\
\hline FT & 0.996 & $(0.993-1.000)$ & 0.046 & & & \\
\hline IVRT & 0.984 & $(0.969-1.000)$ & 0.047 & & & \\
\hline PAAT & 0.972 & $(0.952-0.993)$ & 0.008 & & & \\
\hline Septal MAPSE & 0.235 & $(0.059-0.939)$ & 0.040 & & & \\
\hline E/e' & 1.090 & $(1.009-1.177)$ & 0.029 & & & \\
\hline Septal a' & 0.787 & $(0.632-0.981)$ & 0.033 & & & \\
\hline Septal s' & 0.663 & $(0.447-0.983)$ & 0.041 & & & \\
\hline \multicolumn{7}{|l|}{ HFpEF patients } \\
\hline MLHFQ - total score & 1.080 & $(1.032-1.131)$ & $<0.001$ & 1.137 & $(1.036-1.249)$ & 0.007 \\
\hline MLHFQ - physical score & 1.088 & $(1.010-1.173)$ & 0.026 & & & \\
\hline MLHFQ - emotional score & 1.213 & (1.055-1.396) & 0.007 & & & \\
\hline Diabetes mellitus & 3.556 & $(1.089-11.61)$ & 0.036 & 26.88 & $(1.791-400.8)$ & 0.017 \\
\hline Hemoglobin & 0.711 & $(0.514-0.983)$ & 0.039 & & & \\
\hline NYHA class & 3.038 & $(1.271-7.262)$ & 0.012 & & & \\
\hline BSA & 0.003 & $(0.000-0.241)$ & 0.009 & & & \\
\hline LVMI & 10.50 & $(1.007-1.096)$ & 0.023 & & & \\
\hline Lateral a' & 0.768 & $(0.604-0.978)$ & 0.032 & & & \\
\hline Lateral s' & 0.468 & $(0.258-0.850)$ & 0.013 & & & \\
\hline \multicolumn{7}{|l|}{ HFrEF patients } \\
\hline MLHFQ - physical score & 1.076 & (1.002-1.156) & 0.044 & & & \\
\hline Age & 1.071 & $(1.013-1.132)$ & 0.015 & 1.113 & $(1.024-1.209)$ & 0.012 \\
\hline NYHA class & 2.501 & $(1.064-5.881)$ & 0.036 & & & \\
\hline LVM & 1.010 & $(1.001-1.019)$ & 0.036 & 1.015 & $(1.000-1.030)$ & 0.047 \\
\hline LA diameter & 3.183 & $(1.356-7.475)$ & 0.008 & 7.401 & $(1.821-30.08)$ & 0.005 \\
\hline LV EDD & 1.070 & $(1.002-1.143)$ & 0.044 & & & \\
\hline
\end{tabular}

MLHFQ Minnesota living with heart failure questionnaire, NYHA New York Heart Association, BSA body surface area, $L V$ left ventricle, $L V M$ left ventricular mass, $L A$ left atrium, EDD end-diastolic dimension, $F T$ filling time, $I V R T$ isovolemic relaxation time, MAPSE mitral annular plane systolic excursion, $E$ early diastolic filling, velocity, $e^{\prime}$ early diastolic myocardial velocity, s' systolic myocardial velocity, PAAT pulmonary artery acceleration time, LVMI left ventricular mass index, HFrEF heart failure with reduced ejection fraction

Furthermore, patients with limited exercise capacity proved to have dilated LA [46, 47], the underlying pathophysiology of which is known to be complicated. It proved to be related to the high filling pressures in some [48] and poor LA emptying, as shown be short LV filling time, in others [49]. In addition to the variety of mechanisms of disturbed physiology, the matter is further complicated by the way patients differ in their response to treatment.
While the former group usually responds to LA pressure lowering medications i.e. ACE-inhibitors or A2 blockers [50], the latter respond better to cardiac resynchronization therapy [51]. Finally, it seems that predictors of the limited exercise capacity differed fundamentally according to the cardiac physiology. While specifically the causes of LA enlargement; pressure, mitral regurgitation, stiff LV, etc., that limited patients exercise in HFrEF, the respective reasons 


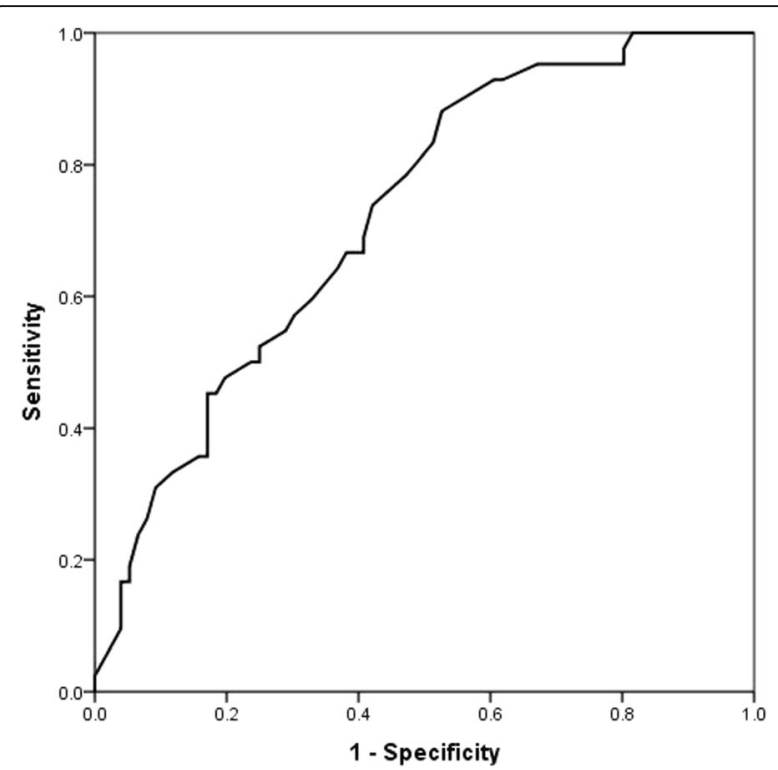

Fig. 2 ROC-curve of MLHFQ - total score in predicting poor exercise performance on 6-min walk test in patients with heart failure

were multifactorial including age, diabetes, as well as emotional and physical scores that predicted exercise capacity in HFpEF. The latter finding adheres to what is known about HFpEF in terms of its etiology, comorbidities as well as limited benefit when using conventional guidelinesbased treatment recommendations [52]. The lack of an acceptable relationship between LA volume and exercise capacity in HFpEF could be explained by either strict early treatment with vasodilators which reduced cavity pressure and hence volume or less myocardial stiffness compared with HFrEF. Also, despite higher AF prevalence in HFrEF patients compared to HFpEF, our analysis suggest that AF was not necessarily a determinant factor for the difference in relationship between left atrial enlargement and 6MWT. It seems therefore that more than one factor could contribute to the lack of direct relationship between the LA volume and exercise capacity in HFpEF. It was however not feasible to run a number of permutations and combinations in order to identify the additive value of various individual variables in predicting exercise capacity.

\section{Clinical implications}

Our findings suggest that the MLHFQ correlates with $6 \mathrm{MWT}$ distance in heart failure patients as a whole and is able, fairly accurately, to predict those with limited exercise capacity. These findings apply better to patients with HFpEF much more than those with HFrEF in whom clearly signs of raised LA pressures are those which independently determine their limited exercise capacity. These differences support the need for continuing the use of detailed Doppler echocardiographic follow up of heart failure patients in order to better understand the pattern of disturbances that explain symptoms as well as the most accurate treatment option.

\section{Limitations}

Obvious limitations can easily be seen in this study. The small number of patients included in this study limits general application of the findings before results are revalidated in a larger cohort. We consider that further prospective cohort studies with a larger sample size, are undoubtedly needed to strengthen or refute our findings. Speckle tracking ultrasonography to measure the global longitudinal strain, which might be associated with reduced functional capacity in HF patients was not used. However, assessing longitudinal LV function with conventional tools, provided an estimate of other overall longitudinal LV function. We cannot ignore the emotional element in conducting the 6-MWT and patient encouragement to walk faster, although unassisted. We did not assess the reproducibility of the results of the MLHFQ neither the 6-MWT distance, which could have shown significant differences.

\section{Conclusion}

Although the conventionally used MLHFQ, irrespective of its components, correlates closely with the 6-MWT distance in HF patients particularly HFpEF. Raised filling pressures seem to be the strongest independent predictor of limited exercise capacity in HFrEF. These differences might impact treatment options in the two conditions.

\section{Additional file}

Additional file 1: Table S1. The Minnesota Living with Heart Failure Questionnaire. (DOC 29 kb)

\section{Abbreviations}

6-MWT: Minute walk test; A: Atrial diastolic velovity; a': Atrial myocardial velocity; BMI: Body mass index; BSA: Body surface area; DT: Deceleration time; E: Early diastolic velocity; e': Early diastolic myocardial velocity; EF: Ejection fraction; FT: Filling time; HF: Heart failure; HFpEF: Heart failure with preserved ejection fraction; HFrEF: Heart failure with reduced ejection fraction; LA EF: Left atrial emptying fraction; LA: Left atrium; LAV max: Left atrial maximal volume; LAV min: Left atrial minimal volume; LV: Left ventricle; LVM: Left ventricular mass; LVMI: Left ventricular mass index; LVPWd: Left ventricular posterior wall in diastole; MAPSE: Mitral annular plane systolic excursion; MLHFQ: The Minnesota Living with Heart Failure Questionnaire; NYHA: New York Heart Association; PA: Pulmonary artery; QoL: Quality of life; RV: Right ventricle; s': Systolic myocardial velocity; TAPSE: Tricuspidal annular plane systolic excursion

\section{Acknowledgements}

Not applicable.

Funding

Not applicable.

Availability of data and materials

The datasets used and analyzed during the current study are available from the corresponding author on reasonable request. 


\section{Authors' contributions}

$\mathrm{GB}$, SE and $\mathrm{MH}$ designed the manuscript; Data acquisition was made by $A B$, $A P$ and $E H ; A A, P I$ and $G B$ analyzed and interpreted the data; $A B, A A$ AP and EH drafted the manuscript; $\mathrm{MH}, \mathrm{PI}, \mathrm{GB}$ and SE revised critically. All authors read, approved the manuscript for publication and agreed to be authors of the manuscript

\section{Ethics approval and consent to participate}

All patients signed a written informed consent to participate in the study, which was approved by the Ethics Committee of the Medical Faculty, University of Prishtina (Statement Nr.3729, date 22.10.2010). This study was supported and monitored by Kosovo Society of Cardiology [27], which is trying to implement European Society of Cardiology guidelines and other current diagnostic and therapeutic recommendations.

\section{Consent for publication}

Not applicable.

\section{Competing interests}

The authors declare that they have no competing interests.

\section{Publisher's Note}

Springer Nature remains neutral with regard to jurisdictional claims in published maps and institutional affiliations.

\section{Author details}

${ }^{1}$ Clinic of Cardiology, University Clinical Centre of Kosova, Rrethi i Spitalit, P.N, 10000 Prishtina, Kosovo. ${ }^{2}$ Medical Faculty, University of Prishtina, Prishtina, Kosovo. ${ }^{3}$ Department of Public Health and Clinical Medicine, Umeå University and Heart Centre, Umeå, Sweden. ${ }^{4}$ Molecular \& Clinical Sciences Research Institute, St George University London, London, UK.

Received: 21 August 2017 Accepted: 13 October 2017

Published online: 17 October 2017

\section{References}

1. Jessup M, Marwick TH, Ponikowski P, Voors AA, Yancy CW. 2016 ESC and ACC/AHA/HFSA heart failure guideline update - what is new and why is it important? Nat Rev Cardiol. 2016;13(10):623-8.

2. Working Group on Cardiac Rehabilitation \& Excercise Physiology and Working Group on Heart Failure of the European Society of Cardiology. Recommendations for exercise testing in chronic heart failure patients. Eur Heart J. 2001:22(1):37-45.

3. Wilson JR, Hanamanthu S, Chomsky DB, Davis SF. Relationship between exertional symptoms and functional capacity in patients with heart failure. Am Coll Cardiol. 1999;33(7):1943-7.

4. Bittner V, Weiner DH, Yusuf S, Rogers WJ, Mclntyre KM, Bangdiwala SI, et al. Prediction of mortality and morbidity with a 6-min walk test in patients with left ventricular dysfunction. SOLVD investigators. JAMA. 1993;270(14):1702-7.

5. Alahdab MT, Mansour IN, Napan S, Stamos TD. Six minute walk test predicts long-term all-cause mortality and heart failure Rehospitalization in AfricanAmerican patients hospitalized with acute Decompensated heart failure. J Card Fail. 2009;15(2):130-5.

6. Morcillo C, Aguado O, Delás J, Rosell F. Utilidad del Minnesota Living With Heart Failure Questionnaire en la evaluación de la calidad de vida en enfermos con insuficiencia cardiaca. Rev Esp Cardiol. 2007;60(10):1093-6.

7. Bussoni MF, Guirado GN, Roscani MG, Polegato BF, Matsubara LS, Bazan SGZ, et al. Diastolic function is associated with quality of life and exercise capacity in stable heart failure patients with reduced ejection fraction. Braz J Med Biol Res Rev Bras Pesqui Médicas E Biológicas Soc Bras Biofísica Al. 2013;46(9):803-8.

8. Ponikowski P, Voors AA, Anker SD, Bueno H, Cleland JGF, Coats AJS, et al. 2016 ESC guidelines for the diagnosis and treatment of acute and chronic heart failure: the task force for the diagnosis and treatment of acute and chronic heart failure of the European Society of Cardiology (ESC) developed with the special contribution of the heart failure association (HFA) of the ESC. Eur Heart J. 2016;37(27):2129-200.

9. Wasywich CA, Gamble GD, Whalley GA, Doughty RN. Understanding changing patterns of survival and hospitalization for heart failure over two decades in New Zealand: utility of "days alive and out of hospital" from epidemiological data. Eur J Heart Fail. 2010;12(5):462-8.

10. Joffe SW, Webster K, McManus DD, Kiernan MS, Lessard D, Yarzebski J, et al. Improved survival after heart failure: a community-based perspective. J Am Heart Assoc. 2013;2(3):e000053.

11. Stevenson LW. Design of therapy for advanced heart failure. Eur J Heart Fail. 2005;7(3):323-31.

12. Jaarsma $T$, Beattie JM, Ryder M, Rutten FH, McDonagh T, Mohacsi $P$, et al. Palliative care in heart failure: a position statement from the palliative care workshop of the heart failure Association of the European Society of cardiology. Eur J Heart Fail. 2009;11(5):433-43.

13. Hoekstra T, Lesman-Leegte I, van Veldhuisen DJ, Sanderman R, Jaarsma T. Quality of life is impaired similarly in heart failure patients with preserved and reduced ejection fraction. Eur J Heart Fail. 2011;13(9):1013-8.

14. Rector TS, Cohn JN. Assessment of patient outcome with the Minnesota living with heart failure questionnaire: reliability and validity during a randomized, double-blind, placebo-controlled trial of pimobendan. Pimobendan multicenter research group. Am Heart J. 1992;124(4):1017-25.

15. Dobre D, de Jongste MJL, Haaijer-Ruskamp FM, Sanderman R, van Veldhuisen DJ, Ranchor AV. The enigma of quality of life in patients with heart failure. Int J Cardiol. 2008:125(3):407-9.

16. Guyatt GH. Measurement of health-related quality of life in heart failure. J Am Coll Cardiol. 1993;22(4):A185-91.

17. Ahmeti A, Bajraktari G. Use of the "Minnesota living with heart failure questionnaire" quality of life questionnaire in Kosovo's heart failure patients. Int Cardiovasc Forum J. 2016;9(1):36-40.

18. Clark DO, Tu W, Weiner M, Murray MD. Correlates of health-related quality of life among lower-income, urban adults with congestive heart failure. Heart Lung J Acute Crit Care. 2003;32(6):391-401.

19. Gott M. Predictors of the quality of life of older people with heart failure recruited from primary care. Age Ageing. 2006;35(2):172-7.

20. Pascale JM, Sosa CE, Orn A. Evaluation of interleukin-2 soluble receptor in patients with trypanosomiasis americana in Panama. Rev Med Panama. 1991;16(3):184-8.

21. Adebayo S, Olunuga T, Durodola A, Ogah O. Quality of life in heart failure: a review. Niger. J Cardiol. 2017;14(1):1.

22. Hoekstra T, Jaarsma T, van Veldhuisen DJ, Hillege HL, Sanderman R, LesmanLeegte I. Quality of life and survival in patients with heart failure. Eur J Heart Fail. 2013;15(1):94-102.

23. Mbakwem AC, Aina FO, Amadi CE, Akinbode AA, Mokwunyei J. Comparative analysis of the quality of life of heart failure patients in south western Nigeria. World J Cardiovasc Dis. 2013;3(1):146-53.

24. Juenger J, Schellberg D, Kraemer S, Haunstetter A, Zugck C, Herzog W, et al. Health related quality of life in patients with congestive heart failure: comparison with other chronic diseases and relation to functional variables. Heart Br Card Soc. 2002:87(3):235-41.

25. dos SJJA, JEA P, PRS B. Quality of life and clinical indicators in heart failure: a multivariate analysis. Arq Bras Cardiol. 2009;93(2):159-66.

26. Bajraktari G, Batalli A, Poniku A, Ahmeti A, Olloni R, Hyseni V, et al. Left ventricular markers of global dyssynchrony predict limited exercise capacity in heart failure, but not in patients with preserved ejection fraction. Cardiovasc Ultrasound. 2012;10(1):36.

27. Bajraktari G. The Kosovo Society of Cardiology. Int Cardiovasc Forum J [Internet]. 201512 [cited 2016 Sep 18];2(1). Available from: http://icfjournal. org/icfj/index.php/icfj/article/view/93.

28. Gardin JM, Adams DB, Douglas PS, Feigenbaum H, Forst DH, Fraser AG, et al. Recommendations for a standardized report for adult transthoracic echocardiography: a report from the American Society of Echocardiography's nomenclature and standards committee and task force for a standardized echocardiography report. J Am Soc Echocardiogr Off Publ Am Soc Echocardiogr. 2002;15(3):275-90.

29. Lang RM, Bierig M, Devereux RB, Flachskampf FA, Foster E, Pellikka PA, et al. Recommendations for chamber quantification. Eur J Echocardiogr J Work Group Echocardiogr Eur Soc Cardiol. 2006:7(2):79-108.

30. Devereux RB, Alonso DR, Lutas EM, Gottlieb GJ, Campo E, Sachs I, et al. Echocardiographic assessment of left ventricular hypertrophy: comparison to necropsy findings. Am J Cardiol. 1986;57(6):450-8.

31. Höglund C, Alam M, Thorstrand C. Atrioventricular valve plane displacement in healthy persons. An echocardiographic study. Acta Med Scand. 1988;224(6):557-62.

32. Wakatsuki Y, Funabashi N, Mikami Y, Shiina Y, Kawakubo M, Takahashi M, et al. Left atrial compensatory function in subjects with early stage primary 
hypertension assessed by using left atrial volumetric emptying fraction acquired by transthoracic echocardiography. Int J Cardiol. 2009;136(3):363-7.

33. Henein MY, Das SK, O'Sullivan C, Kakkar W, Gillbe CE, Gibson DG. Effect of acute alterations in afterload on left ventricular function in patients with combined coronary artery and peripheral vascular disease. Heart $\mathrm{Br}$ Card Soc. 1996;75(2):151-8.

34. Duncan AM, Francis DP, Henein MY, Gibson DG. Importance of left ventricular activation in determining myocardial performance (Tei) index: comparison with total isovolumic time. Int J Cardiol. 2004;95(2-3):211-7.

35. Tei C, Ling LH, Hodge DO, Bailey KR, JK O, Rodeheffer RJ, et al. New index of combined systolic and diastolic myocardial performance: a simple and reproducible measure of cardiac function-a study in normals and dilated cardiomyopathy. J Cardiol. 1995;26(6):357-66.

36. Zoghbi WA, Enriquez-Sarano M, Foster E, Grayburn PA, Kraft CD, Levine RA, et al. Recommendations for evaluation of the severity of native valvular regurgitation with two-dimensional and Doppler echocardiography. J Am Soc Echocardiogr Off Publ Am Soc Echocardiogr. 2003;16(7):777-802.

37. Olariu A, Wellnhofer E, Gräfe M, Fleck E. Non-invasive estimation of left ventricular end-diastolic pressure by pulmonary venous flow deceleration time. Eur J Echocardiogr J Work Group Echocardiogr Eur Soc Cardiol. 2003;4(3):162-8.

38. Mowat DH, Haites NE, Rawles JM. Aortic blood velocity measurement in healthy adults using a simple ultrasound technique. Cardiovasc Res. 1983; 17(2):75-80.

39. Finegold JA, Manisty CH, Cecaro F, Sutaria N, Mayet J, Francis DP. Choosing between velocity-time-integral ratio and peak velocity ratio for calculation of the dimensionless index (or aortic valve area) in serial follow-up of aortic stenosis. Int J Cardiol. 2013;167(4):1524-31.

40. Guyatt GH, Sullivan MJ, Thompson PJ, Fallen EL, Pugsley SO, Taylor DW, et al. The 6-min walk: a new measure of exercise capacity in patients with chronic heart failure. Can Med Assoc J. 1985;132(8):919-23.

41. Guyatt GH, Thompson PJ, Berman LB, Sullivan MJ, Townsend M, Jones NL, et al. How should we measure function in patients with chronic heart and lung disease? J Chronic Dis. 1985;38(6):517-24.

42. Ingle L, Rigby AS, Nabb S, Jones PK, Clark AL, Cleland JGF. Clinical determinants of poor 6-min walk test performance in patients with left ventricular systolic dysfunction and no major structural heart disease. Eur J Heart Fail. 2006;8(3):321-5.

43. Kotecha D, Flather MD, Altman DG, Holmes J, Rosano G, Wikstrand J, et al. Heart rate and rhythm and the benefit of Beta-blockers in patients with heart failure. J Am Coll Cardiol. 2017:69(24):2885-96.

44. Roth S, Fernando C, Azeem S, Moe GWI. There a role for Ivabradine in the contemporary Management of Patients with chronic heart failure in academic and community heart failure clinics in Canada? Adv Ther. 2017; 34(6):1340-8.

45. Hidalgo FJ, Anguita M, Castillo JC, Rodríguez S, Pardo L, Durán E, et al. Effect of early treatment with ivabradine combined with beta-blockers versus beta-blockers alone in patients hospitalised with heart failure and reduced left ventricular ejection fraction (ETHIC-AHF): a randomised study. Int J Cardiol. 2016 Aug 15:217:7-11.

46. Acarturk E, Koc M, Bozkurt A, Unal I. Left atrial size may predict exercise capacity and cardiovascular events in patients with heart failure. Tex Heart Inst J. 2008:35:136-43.

47. Batalli A, lbrahimi P, Bytyi? I, Ahmeti A, Haliti E, Elezi S, et al. Different determinants of exercise capacity in HFpEF compared to HFrEF. Cardiovasc Ultrasound [Internet]. 2017 Dec [cited 2017 Jun 15];15(1). Available from: http:// cardiovascularultrasound.biomedcentral.com/articles/10.1186/s12947-017-0103-x.

48. Bajraktari G, Dini FL, Fontanive P, Elezi S, Berisha V, Napoli AM, et al. Independent and incremental prognostic value of Doppler-derived left ventricular total isovolumic time in patients with systolic heart failure. Int J Cardiol. 2011;148(3):271-5.

49. Bytyçi I, Bajraktari G, Ibrahimi P, Berisha G, Rexhepaj N, Henein MY. Left atrial emptying fraction predicts limited exercise performance in heart failure patients. IJC. Heart Vessel. 2014;4:203-7.

50. Henein MY, O'Sullivan CA, Coats AJ, Gibson DG. Angiotensin-converting enzyme (ACE) inhibitors revert abnormal right ventricular filling in patients with restrictive left ventricular disease. J Am Coll Cardiol. 1998;32(5):1187-93.
51. Salukhe TV, Francis DP, Morgan M, Clague JR, Sutton R, Poole-Wilson P, et al. Mechanism of cardiac output gain from cardiac resynchronization therapy in patients with coronary artery disease or idiopathic dilated Cardiomyopathy. Am J Cardiol. 2006;97(9):1358-64.

52. Oktay A, Shah S. Diagnosis and Management of Heart Failure with preserved ejection fraction: 10 key lessons. Curr Cardiol Rev. 2014;11(1):42-52.

\section{Submit your next manuscript to BioMed Central and we will help you at every step:}

- We accept pre-submission inquiries

- Our selector tool helps you to find the most relevant journal

- We provide round the clock customer support

- Convenient online submission

- Thorough peer review

- Inclusion in PubMed and all major indexing services

- Maximum visibility for your research

Submit your manuscript at www.biomedcentral.com/submit
Biomed Central 